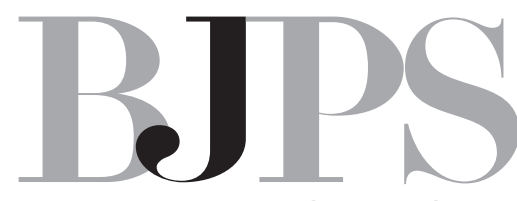

\title{
BJPS as strategical for Pharmaceutical Sciences
}

QUALIS has been used by the Committee for Improvement of Higher Level Personnel (CAPES) as a tool for stratifying the quality of the intellectual production of Graduate Programs allover Brazil. It has dictating the profile of the areas of knowledge in terms of publications. Although polemic in some aspects, its application intends to reflect how and where the diverse areas divulge its scientific production. The criteria for classifying the journals depend on the area and have been periodically reviewed.

For QUALIS 2012 version, CAPES asked the areas to choose journals considered strategic for their development. The journals most cited by researchers and graduate students should translate their importance to the areas. So, BJPS, due to the high number of citations - more than 190 - during the last triennium (2007-2009) of evaluation was indicated as one of the strategic journals. CAPES objective is also to support those nominated journals, which is expected. Strategically, its stratification should be changed to higher levels and based on this decision, BJPS moved from B3 to B2, independently of the Pharmacy Area indexing criteria.

This distinction emphasizes BJPS as the main scientific vehicle for divulging the Pharmaceutical Sciences produced in the country. A higher number of manuscript submissions is thus expected. This generates more responsibilities and foster BJPS Scientific, Associate and Executive Editors to look forward initiatives toward improvement. Besides, the initial impact index attributed to BJPS by JCR 2010 should increase.

It is worth to note that BJPS is in the process of change from an exclusively national to an international profile, especially after its indexing by ISI Thomas Reuters. Asian, African and Latin American scientific communities are increasingly submitting their manuscripts to be published in the journal. We truly value their collaboration and expect to reach other communities, as well.

Starting the volume 48, we are willing to face the challenges and direct our efforts to raise BJPS to the top journals of Pharmaceutical Sciences. We thank the authors and editors in advance for precious collaboration.

Elizabeth Igne Ferreira

Scientific Editor 